\title{
Distribution and clinical relevance of phospholipids in hepatocellular carcinoma
}

\author{
Zhirong Liu ${ }^{1,4} \cdot$ Zhen Zhang ${ }^{1} \cdot \mathrm{Hao} \mathrm{Mei}^{2} \cdot$ Jinghe $\mathrm{Mao}^{3} \cdot$ Xinchun Zhou $^{4}$
}

Received: 9 December 2019 / Accepted: 21 March 2020 / Published online: 5 June 2020

(c) The Author(s) 2020

\begin{abstract}
Background Hepatocellular carcinoma (HCC) is the most common liver cancer and featured with prominent disparity in incidence and mortality rate between male and female. It remains unclear whether alterations of phospholipids (PL) in hepatic tissues contribute to the pathogenesis, progression, and disparity of HCC.

Methods Using electrospray ionization mass spectrometry (ESI-MS), PL profiles including 320 individual phospholipid species in 13 PL classes were determined in paired samples from HCC and adjacent benign hepatic tissues (BHT).

Results (1) Concentrations of PLs in most of individual species, in subgroups and in total were decreased in HCC than in BHT in all studied population; (2) the number of individual PL species significantly different between HCC and BHT, and the number of PLs in six subgroups and in total decreased in HCC were more in male population than in female population; (3) panels of PL parameters (more in male population than in female population) were identified as biomarkers in differentiation of HCC from BHT, and in the prediction of pathological grade and clinical stage of HCC with high sensitivity, specificity, and accuracy.

Conclusion It is concluded that alterations of PLs in hepatic tissues play important roles in pathogenesis, progression, and gender disparity of HCC.
\end{abstract}

Keywords Hepatocellular carcinoma (HCC) · Phospholipids (PL) · Lipidomics · Gender · Race · Disparity · Biomarkers · Benign hepatic tissues (BHT) · African American (AA) · Caucasian American (CA)

\section{Introduction}

Hepatocellular carcinoma (HCC) is a primary malignancy of liver with high morbidity and mortality worldwide [1]. HCC is known to be gender disparate, being more frequently

Electronic supplementary material The online version of this article (https://doi.org/10.1007/s12072-020-10056-8) contains supplementary material, which is available to authorized users.

Xinchun Zhou

xzhou@umc.edu

1 Department of Biochemistry, Shanxi Medical University, Shanxi Province, Taiyuan 030001, China

2 Department of Computer Sciences, University of Mississippi Medical Center, Jackson, MS 39216, USA

3 Department of Biology, Tougaloo College, Tougaloo, MS 39174, USA

4 Department of Pathology, University of Mississippi Medical Center, Jackson, MS 39216, USA occurred and aggressive in male than in female, accounting for $7.5 \%$ of total male cancers, and $3.4 \%$ of total female cancers, respectively [2]. The early detection and treatment can increase cure rate of HCC; however, the prognosis is poor for patients at advanced stage of HCC. To date, the molecular mechanisms underlying high morbidity, mortality, and gender disparity of HCC remain unclear.

Phospholipids (PLs) have diverse biological functions in cells. In addition to being building blocks in membranous structures, PLs also play important roles in cellular signaling and cell-cell interactions in tissues. Metabolic alterations of various PLs have been reported to link to cancer proliferation. However, differences in phospholipid profiles have been rarely studied between HCC and benign hepatic tissues (BHT), and not investigated between male and female populations.

Lipidomics is a powerful tool available for the analysis and characterization of lipids and their interacting moieties. Since the developments in mass spectrometry, advanced analytical methods are able to identify and quantify a large 
number of individual species, classes and subgroups of lipids, especially PLs at the same time [3]. Lipid profiling is now widely applied in the determination of lipid alterations between different pathological conditions in various cancers, such as renal clear cell carcinoma and prostate cancer [4, 5]. Regarding the HCC, lipid profiling has been mostly performed on body fluids in the identification of diagnostic biomarkers and in characterization of metabolic pathways for certain PLs. However, global PL profiles have not been performed on and compared between HCC and benign hepatic tissues (BHT). Cotte et al. performed electrospray ionization mass spectrometry (ESI-MS) for seven phospholipid classes on plasma samples from 45 HCC patients and 45 cirrhotic patients without HCC, suggesting that plasma phospholipid profiles related to HCC risk in liver cirrhotic patients, and some phospholipid species might serve as potential biomarkers in predicting HCC patient outcomes [6]. Another study reported that HCC is characterized by reduced ceramide, lower level of polyunsaturated (PUFA) phospholipids, and increased CE and SM [7]. Interestingly, a recent study determined phospholipid profiles in samples from both hepatic tissues and serum from $50 \mathrm{HCC}$ patients using an untargeted lipidomics approach. The results showed that the most phospholipid species showed opposite tendency of changes between patients' HCC tissues and sera, implying that lipid metabolisms are likely to be modulated in different manners in HCC tissues and peripheral circulations [8]. To date, comparison of PL profiles between HCC and adjacent benign hepatic tissues (BHT) from same patients has not been studied; and none of studies has linked the differences in hepatic PL profiles to gender disparity in incidence and mortality rate of HCC.

In the present study, we performed ESI-MS for 320 phospholipid species in 13 phospholipid classes on paired HCC and BHT tissues from 32 male and female HCC patients. Our aims are to determine differences in PL distribution between HCC and BHT, to reveal gender disparity in PL profiles and their clinic relevance between male and female populations, to identify PL biomarkers in the differentiation of HCC from BHT and in the prediction of progression of $\mathrm{HCC}$, and to seek novel therapeutic targets in PL metabolisms for treatment of HCC.

\section{Materials and methods}

\section{Patients and sample collection}

This study was approved by the Institutional Review Board of the University of Mississippi Medical Center. The study cohort comprised 32 patients with clinical HCC. A total of 64 samples, including $32 \mathrm{HCC}$ tissues and their accompanied BHT were serially collected by the Cooperative
Human Tissue Network (CHTN). The written consents were obtained from patients prior to the donation of tissue samples. To insure quality and quantity of samples, each sample should be collected within 10 years and weighted $>50 \mathrm{mg}$. The diagnosis of each HCC and BHT sample was reconfirmed by the pathologists at sites patients were enrolled in. Histological features for BHT samples included normal liver tissues, inflammation/hepatitis, alcoholic fatty liver disease (AFLD), non-alcoholic fatty liver disease (NAFLD), and cirrhosis, but none of BHT samples contains liver cancer cells. Because the numbers in each of the categories with benign hepatic changes were limited, all non-cancer tissues adjacent to HCC were grouped as benign hepatic tissues (BHT). None of the identifiable information, such as patient's name, date of birth, and contact information was provided. The geographic, clinical, and pathological information is listed in Table 1.

\section{Lipid extraction}

Extraction of total lipids from HCC and BHT tissues was performed with chloroform and methanol, following a modified Bligh and Dyer protocol [9]. Briefly, 85-120 mg tissues was weighed and homogenized. To 0.8 part (volume) aqueous homogenized tissue, 1 part chloroform and 2 parts methanol were added and shaken well, followed by the

Table 1 Patient's geographic and clinic information

\begin{tabular}{ll}
\hline Features & $n(\%)$ \\
\hline Age $(n /$ mean \pm SD) & $32(65.5 \pm 13.8)$ \\
Race & 32 \\
CA & $26(81.25)$ \\
Others & $6(18.75)$ \\
Gender & 32 \\
Male & $21(65.63)$ \\
Female & $11(34.27)$ \\
Pathological grade & $28^{\mathrm{b}}$ \\
Low grade $($ LG) & 11 \\
High grade $(\mathrm{HG})$ & 17 \\
Clinical stage & $28^{\mathrm{a}}$ \\
Early stage (ES) & 20 \\
Late stage (LS) & 8 \\
Margin & $28^{\mathrm{a}}$ \\
Positive & 3 \\
Negative & 25 \\
Lymphovascular invasion & $27^{\mathrm{b}}$ \\
Positive & 12 \\
Negative & 15 \\
\hline
\end{tabular}

${ }^{\mathrm{a}}$ Lack of data on tumor grade, clinical stage and surgical margin in four cases

${ }^{\mathrm{b}} 1$ Lack of data on lymphovascular invasion in five cases 
addition of 1 part chloroform and 1 part water. The sample was shaken well, centrifuged at $3000 \mathrm{rpm}$ for $5 \mathrm{~min}$, and the lower layer was transferred to a glass vial. Then 1 part chloroform was added, the samples were shaken well, and centrifuged at $3000 \mathrm{rpm}$ for $5 \mathrm{~min}$, and the lower layer was transferred to the same glass vial; this process was repeated one more time. The lipid extract solvent collected in the glass vial was evaporated with liquid nitrogen, capped with a Teflon-lined cap, and transported to the KLRC Analytical Laboratory on dry ice for analysis.

\section{Protein extraction and quantification}

Weighed fresh-frozen HCC or BHT tissues were minced and homogenized in RIPA buffer (50 mM Tris-Cl, pH 8.0, $150 \mathrm{mM} \mathrm{NaCl}, 1 \% \mathrm{NP}-40,0.5 \%$ sodium deoxycholate, $0.1 \%$ SDS) with protease inhibitor. The lysates were centrifuged, and the supernatants were collected and stored at $-80{ }^{\circ} \mathrm{C}$. Protein concentration $(\mathrm{mg} /$ wet weight tissues, wwt) was determined with the Pierce $\AA$ BCA Protein Assay Kit (Thermo Fisher Scientific, Wilmington, DE, USA) following the manufacturer's instructions. Briefly, a series of dilutions of bovine serum albumin (BSA) were prepared as a set of protein standards to determine the standard curve. Then the BCA working reagent (WR) was prepared by mixing 50 parts of BCA Reagent A with 1 part of BCA Reagent B (50:1, Reagent A:B). $25 \mu \mathrm{L}$ of HCC or BHT protein was pipetted in replicate into a microplate well and $200 \mu \mathrm{L}$ of the WR was added to each well, and incubated at $37{ }^{\circ} \mathrm{C}$ for $30 \mathrm{~min}$. The quality and quantity of the total isolated proteins were measured and assessed at $570 \mathrm{~nm}$ with the Benchmark microplate reader (Bio-Rad, Hercules, CA, USA).

\section{Electrospray ionization mass spectrometry (ESI-MS)}

The concentrations of PL species were determined by an automated ESI-MS method. Briefly, $35 \mu \mathrm{l}$ lipid extract and internal standard mixture were combined with solvents so that the ratio of chloroform/methanol/300 $\mathrm{mM}$ ammonium acetate in water was $300 / 665 / 35$, and the final volume was $1.2 \mathrm{ml}$. The unfractionated lipid extract in an autosampler vial was introduced by continuous infusion into the ESI source on a triple quadrupole MS/MS (API 4000, Applied Biosystems, Foster City, CA), using an autosampler (LC Mini PAL, CTC Analytics AG, Zwingen, Switzerland) fitted with the required injection loop for the acquisition time and presented to the ESI needle at $30 \mu \mathrm{l} / \mathrm{min}$. PLs were detected by 80 cycles of ESI-MS (MS1) scanning from 150 to 400 $\mathrm{u}$. The source temperature (heated nebulizer) was $100^{\circ} \mathrm{C}$, the interface heater was on, $-4.5 \mathrm{kV}$ was applied to the electrospray capillary, the curtain gas was set at 20 (arbitrary units), and the two ion source gases were set at 45 (arbitrary units). The background of each spectrum was subtracted, the data were smoothed, and peak areas integrated using a custom script and Applied Biosystems Analyst software. The data were isotopically deconvoluted, and the PLs were quantified in comparison to a 15:0 internal standard. Peaks corresponding to the target PLs in these spectra were identified and molar amounts calculated in comparison to the internal standards for PLs. Finally, the data were corrected for the fraction of the sample analyzed and normalized to the sample volume to produce data in the unit of $\mathrm{nmol} / \mathrm{mg}$ wwt HCC or BHT tissues, $\mathrm{nmol} / \mathrm{mg}$ protein, and percentage of each phospholipid species out of total phospholipids according to the number of $\mathrm{nmol} / \mathrm{mg}$ wwt.

\section{Data analysis}

Statistical analysis was carried out in SPSS software (IBM SPSS Statistics 24 software). The mean concentrations of PL parameters between the two groups were compared by independent samples $T$ test. Correlation and/or regression between two series of numerical data was analyzed by bivariate correlation and/or linear regression in Graphpad prism 8 software. Chi-square test was used to compare changes in percentage between two groups. Cluster 3 and Treeview software and programs were used to graph heatmap and hierarchical clustering analysis (HCA). The R software ( Core Team $2016 \mathrm{R}$, https://www.R-project.org) was applied in bioinformatics analyses. The generalized linear model (GLM) with binomial distribution was used to predict disease and control status based on lipid concentration by $\mathrm{R}$ function of GLM. The package of ROCR was used to estimate, sensitivity, specificity, recall, precision, $F$-measure, and area under curve (AUC). Information gain (InfoGain) was determined with simple logistics classification algorithm (a supervised attribute ranking method). The significant $p$ value was set at 0.05 for all results.

\section{Results}

\section{Quality control, PL profiles, data acquisition, and abundance}

To monitor the stability of the detection system and to minimize technical errors originating from sample collection and sample preparation [10], ten repeated aliquots of lipid extracts from pooled hepatic tissues were used as quality controls (QC). QC results demonstrated an acceptable reproducibility of metabolic features and a stability of ESI-MS profiling in 320 out of $335 \mathrm{PL}$ species. The representative spectrums of QC, BHT, and HCC samples are shown in Fig. 1a-c, respectively.

To further prevent errors and to quantitate accurately and reliably, the concentration of each PL species in each 


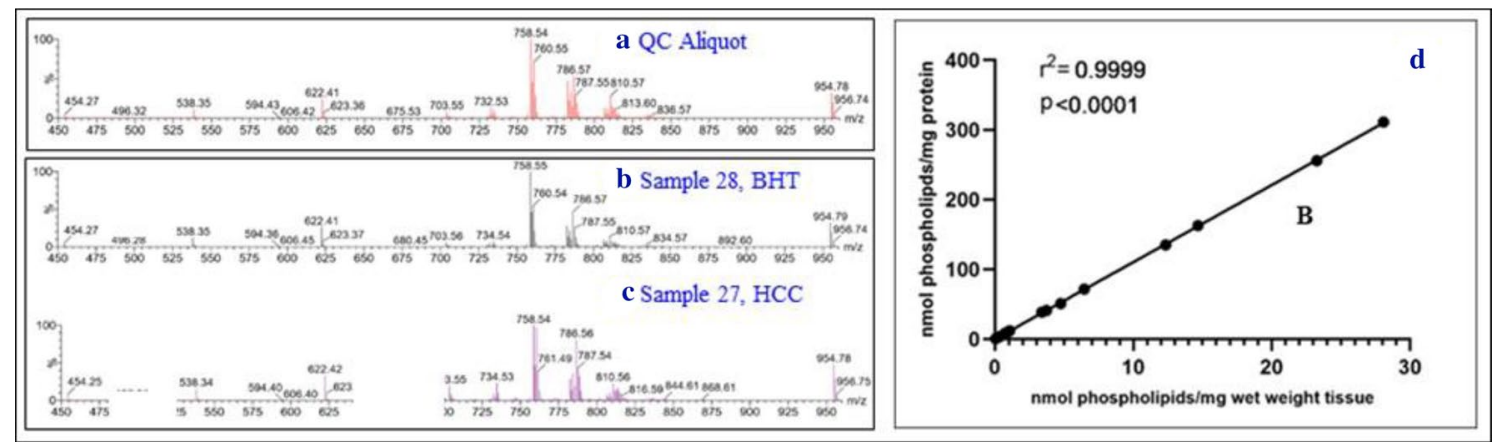

Fig. 1 Representative mass spectra in ESI-MS analysis in the range of $\mathrm{m} / \mathrm{z}$ 450-960 scanning mode for the detection of phospholipid species. a PL spectra represent lipid extract from one of ten aliquot quality control (QC); b PL spectra represent lipid extract from one of benign hepatic tissue (BHT) samples and c PL spectra represent lipid extract from one of hepatocellular carcinoma (HCC) samples;

sample was determined by two methods. One method was to calculate PL concentration in $\mathrm{nmol} / \mathrm{mg}$ wwt freshfrozen hepatic tissue. Another was in $\mathrm{nmol} / \mathrm{mg}$ protein. The correlation and regression analyses were performed on the concentrations of total PLs in 13 classes between two methods. The results indicated that two methods are linearly correlated and this correlation was highly significant $\left(r^{2}=0.9999, p<0.0001\right)$ as shown in Fig. 1d. In this study, the concentrations of PLs were represented by nmol/ mg wwt tissues in data analysis. In addition, the percentage of given PL in individual species or class out of total PLs was used in the calculation of their abundances. Listed in Table 2 are the numbers of individual PL species in 13 PL classes and in six PL subgroups grouped according to similarity of moiety in phosphate head.

The abundances of PLs varied greatly among individual species, classes and subgroups between BHT and HCC. In the level of individual PL species, PC 34:2 was the most abundant among 320 individual PL species and is lower in HCC than in BHT in all population $(-1.6$-fold, $p=0.00010)$, female population $(-1.4$-fold, $p=0.124)$, and male population ( -1.6 -fold, $p=0.003)$. Top ten most abundant individual PL species account for more than $50 \%$ of total PLs in both BHT and HCC in all studied populations. In the level of PL class, among 13 PL classes, PC was the most abundant, accounting for more than $50 \%$ of total PLs, and PE-Cer was the least abundant, accounting for $0.01 \%$ or less of total PLs in both BHT and HCC in all studied populations (Fig. 2).In the level of subgroup, PE-containing PLs was the most abundant among six PL subgroups, accounting for more than 55\% of total PLs in both BHT and HCC in all studied populations. d correlation and regression analyses of mean concentrations in 13 PL classes between two quantitative methods. The plot showed a significantly linear correlation between mean concentrations of $13 \mathrm{PL}$ classes in nmol/mg wet weight tissue (wwt) and that in nmol/mg protein $\left(r^{2}=0.9999, p<0.0001\right)$

Table 2 PL profiles in hepatic tissues

\begin{tabular}{ll}
\hline Phospholipids (PL) & Number of \\
\hline Class & PL species \\
Ceramide phosphorylethanolamine (PE-cer) & 5 \\
Dihydrosphingomyelin (DSM) & 4 \\
Ether-linked phosphatidylcholine (ePC) & 25 \\
Ether-linked phosphatidylethanolamine (ePE) & 27 \\
Ether-linked phosphatidylserine (ePS) & 16 \\
Lysophosphatidylcholine (LPC) & 13 \\
Lysophosphatidylethanolamine (LPE) & 13 \\
Phosphatidic acid (PA) & 22 \\
Phosphatidylcholine (PC) & 46 \\
Phosphatidylethanolamine (PE) & 47 \\
Phosphatidylinositol (PI) & 46 \\
Phosphatidylserine (PS) & 48 \\
Sphingomyelin (SM) & 8 \\
Total phospholipids & 320 \\
Subgroups & \\
Choline-containing PLs (ePC, LPC, PC) & 74 \\
Ethanolamine-containing PLs (PE-Cer, ePE, LPE, PE) & 92 \\
Ether-containing PLs (ePC, ePE, ePS) & 68 \\
Lyso-containing PLs (LPC, LPE) & 26 \\
Serine-containing PLs (ePS, PS) & 64 \\
Sphingomyelin-containing PLs (DSM, SM) & 12 \\
\hline
\end{tabular}

\section{Gender differences of PL concentrations in individual species between BHT and HCC}

The differences of PL concentrations in 320 individual PL species, 13 PL classes, and 6 subgroups between BHT and $\mathrm{HCC}$ are listed in supplemental Tables 1, 2 and 3 for 

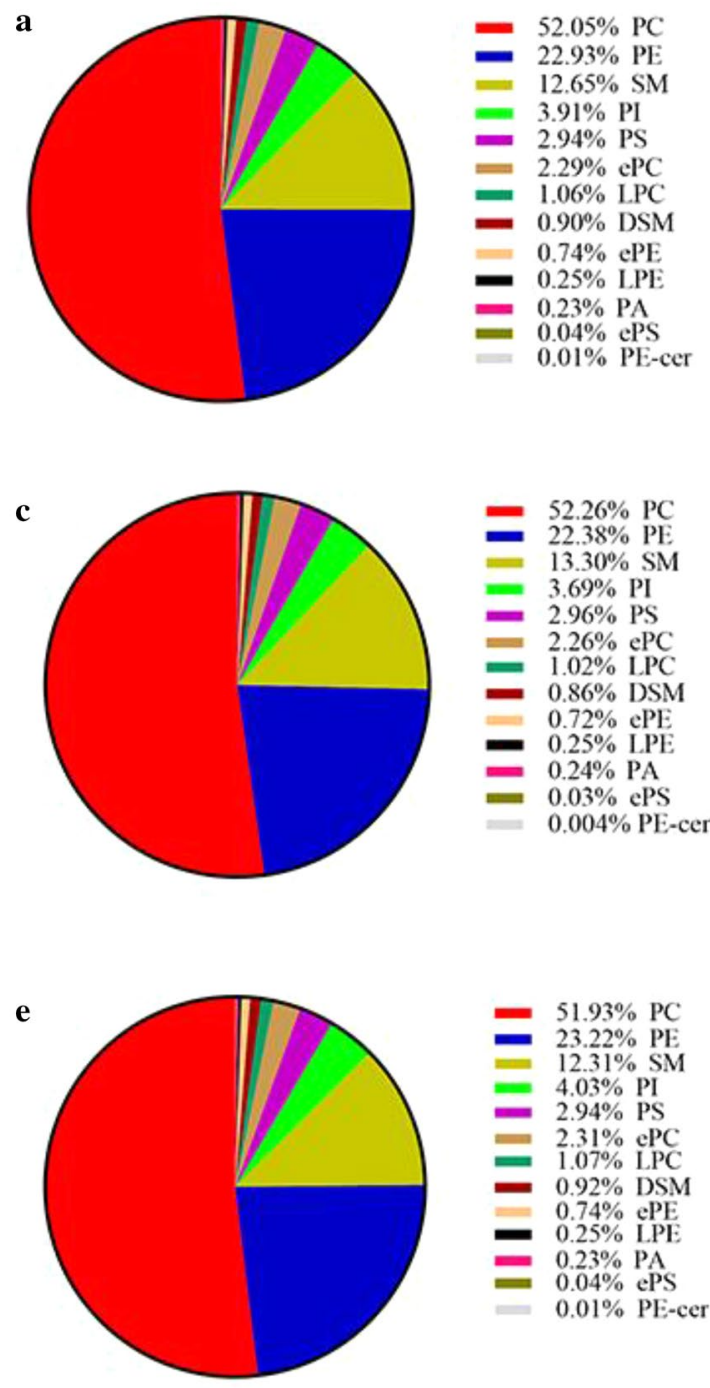

Fig. 2 Abundances of 13 PL classes in BHT and HCC in all studied populations. a BHT and $\mathbf{b} \mathrm{HCC}$ in all population; $\mathbf{c} \mathrm{BHT}$ and $\mathbf{d} \mathrm{HCC}$ in female population; and e BHT and $\mathbf{f} \mathrm{HCC}$ in male population. PC

all population, stratified female population, and stratified male population, respectively.

In the level of individual PL species, 62 out of $320 \mathrm{PL}$ species were significantly different between BHT and HCC in all population. Among them, 21 PL species were significantly increased, and 41 were statistically decreased in HCC than in BHT. The hierarchical cluster analysis (HCA) for the 62 species shown in Fig. 3a revealed that, the 62 individual PL species were scattered in 9 PL classes, among which PC had most (16) individual species, and the rest 8 PL classes had varied number of individual species ranging 1-9. The upper portion in Fig. 3a is predominated with individual PL species having higher concentrations, and the lower portion is predominated with individual PL species having lower concentrations. Interestingly, samples in upper portion have b

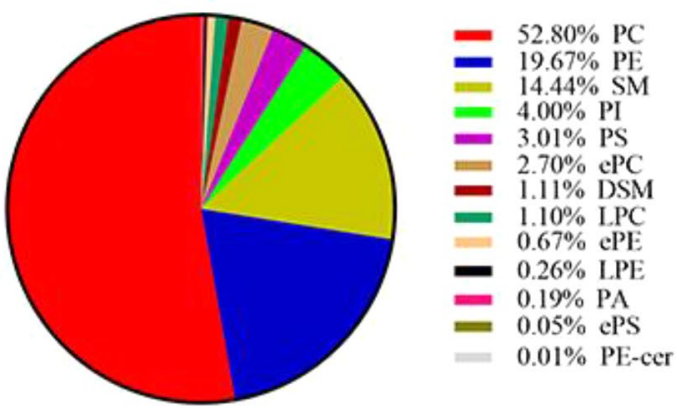

d

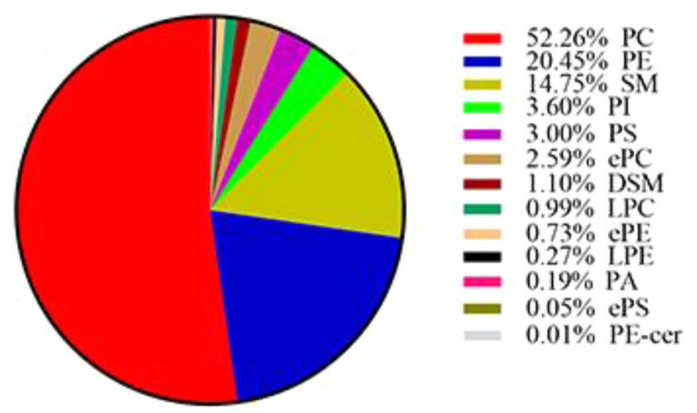

f
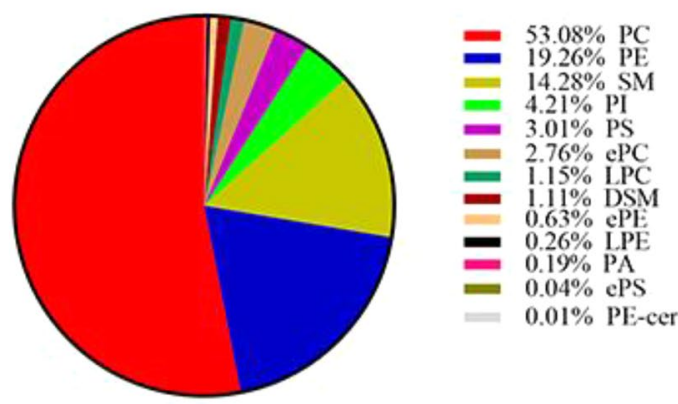

was the most abundant PL class, and PE-Cer was the least abundant PL class in both BHT and HCC in all studied populations

higher percentage of BHT (75\% vs. $31 \%$ ) and are more from patients with older age (67.9 vs. 61.9), lower percentage of females (29.6\% vs. 39.9\%), lower percentage of HCC in late stage (11\% vs. $14 \%$ ), and higher percentage of $\mathrm{HCC}$ in higher grade HCC (76.9\% vs. $46.7 \%)$, as compared to samples in lower portion. Taken together, decrease in individual PL species is seen more in HCC than in BHT, and more related to younger male patients at late stage of $\mathrm{HCC}$, but not to HCC grade in all population.

In female population, significant differences in concentrations between BHT and HCC were only seen in seven PL species: PC (30:0), ePS (38:3), LPC (22:5), ePC (34:0), ePE (34:0), PC (36:1), and PI (42:6) as shown in Fig. 3b. Six out of 7 PL species were significantly higher in HCC than in BHT (raged 1.7-20.6-fold), except LPC (22:5), which was 
Table 3 Differences of phospholipids in class, subgroup, and total between BHT and HCC among populations

\begin{tabular}{|c|c|c|c|c|c|c|}
\hline \multirow[t]{2}{*}{ Phospholipids in class/subgroup/total } & \multicolumn{2}{|c|}{ All population } & \multicolumn{2}{|c|}{ Female population } & \multicolumn{2}{|c|}{ Male population } \\
\hline & $\begin{array}{l}\mathrm{HCC} / \\
\mathrm{BHT} \\
\text { ratio }\end{array}$ & $p$ value & $\begin{array}{l}\mathrm{HCC} / \\
\mathrm{BHT} \\
\text { ratio }\end{array}$ & $p$ value & $\begin{array}{l}\mathrm{HCC} / \\
\mathrm{BHT} \\
\text { ratio }\end{array}$ & $p$ value \\
\hline DSM & 1.0 & 0.9421 & 1.1 & 0.6923 & 1.0 & 0.8285 \\
\hline $\mathrm{ePC}$ & 0.9 & 0.2309 & 0.9 & 0.5192 & 0.9 & 0.3152 \\
\hline $\mathrm{ePE}$ & 0.7 & 0.0157 & 0.8 & 0.4513 & 0.7 & $\mathbf{0 . 0 1 5 6}$ \\
\hline ePS & 0.9 & 0.5439 & 1.2 & 0.6500 & 0.8 & 0.1626 \\
\hline LPC & 0.9 & 0.1927 & 0.8 & 0.4461 & 0.9 & 0.2965 \\
\hline LPE & 0.9 & 0.4605 & 1.0 & 0.9718 & 0.9 & 0.3674 \\
\hline PA & 0.7 & 0.0541 & 0.8 & 0.4782 & 0.7 & 0.0646 \\
\hline $\mathrm{PC}$ & 0.8 & 0.1175 & 0.9 & 0.5516 & 0.8 & 0.1360 \\
\hline PE-Cer & 1.5 & 0.3510 & 2.3 & 0.2497 & 1.2 & 0.6582 \\
\hline PE & 0.7 & 0.0149 & 0.9 & 0.5726 & 0.7 & 0.0073 \\
\hline PI & 0.9 & 0.4207 & 0.9 & 0.8014 & 0.9 & 0.4274 \\
\hline PS & 0.8 & 0.1786 & 0.9 & 0.6498 & 0.8 & 0.1887 \\
\hline SM & 0.9 & 0.3059 & 0.9 & 0.7045 & 0.9 & 0.3225 \\
\hline Choline-containing PLs & 0.8 & 0.1176 & 0.9 & 0.5449 & 0.8 & 0.1379 \\
\hline Ethanolamine-containing PLs & 0.7 & 0.0151 & 0.9 & 0.5713 & 0.7 & 0.0076 \\
\hline Ether-containing PLs & 0.8 & 0.1087 & 0.9 & 0.5014 & 0.8 & 0.1394 \\
\hline Lysol-containing PLs & 0.9 & 0.2198 & 0.9 & 0.5335 & 0.9 & 0.2924 \\
\hline Serine-containing PLs & 0.8 & 0.1801 & 0.9 & 0.6622 & 0.8 & 0.1862 \\
\hline Sphingomyelin-containing PLs & 0.9 & 0.3424 & 0.9 & 0.7466 & 0.9 & 0.3467 \\
\hline Total phospholipids & 0.8 & 0.0813 & 0.9 & 0.5718 & 0.8 & 0.0808 \\
\hline
\end{tabular}

The $p$ value bolded are statistically significant
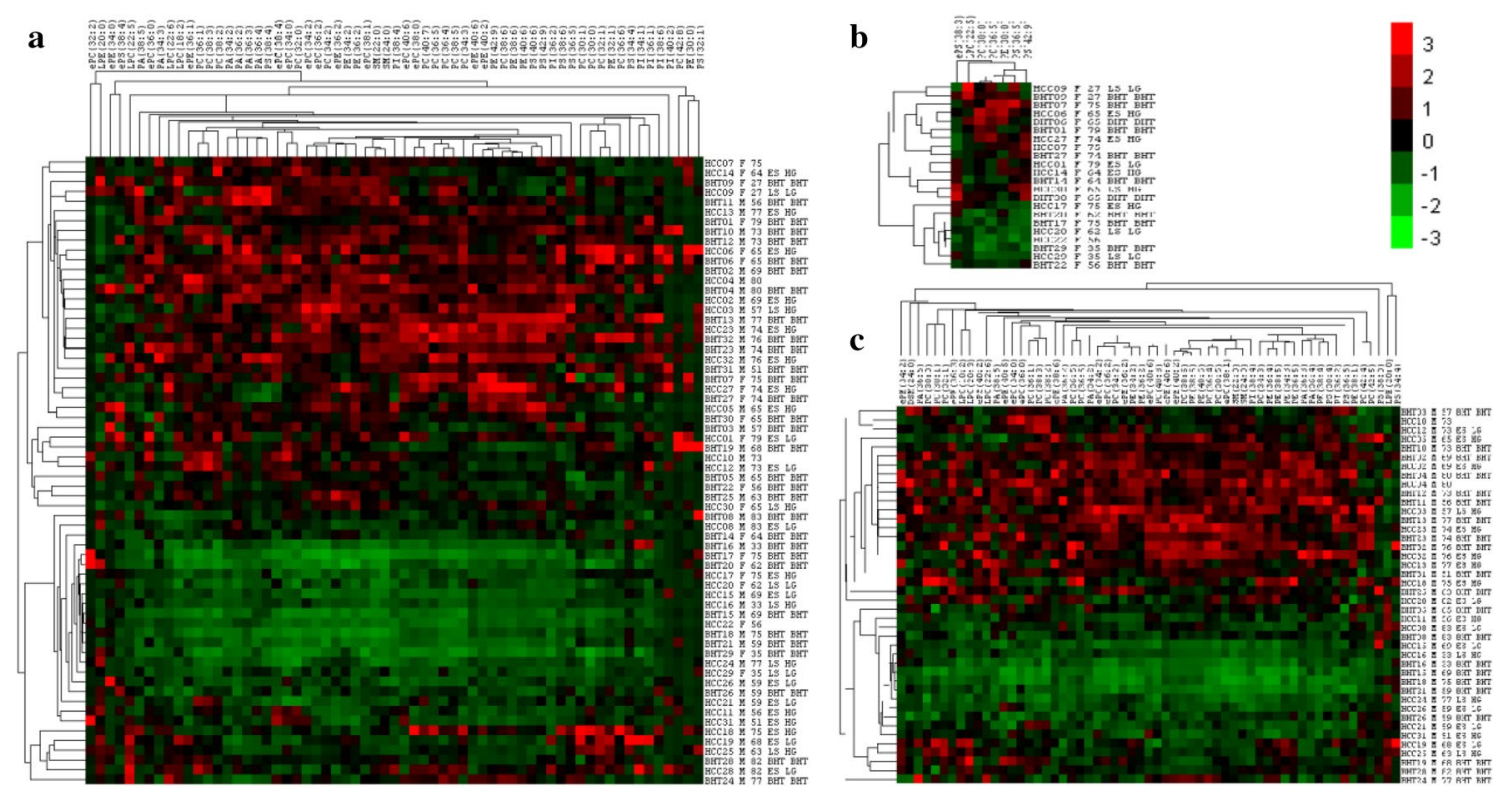

Fig. 3 Heatmap and hierarchical cluster analysis (HCA) for individual PL species that was significantly different between BHT and HCC. The data were normalized by the $Z$ score. The scale bars in color reflect changes in PL concentrations: squares in red represent higher PL concentration, and squares in green represent lower PL concentration. a heatmap and HCA for 62 individual PL species in all population; b heatmap and HCA for seven individual PL species in female population; c heatmap and HCA for 59 individual PL species in male population 
5.7-fold lower in HCC than in BHT. As well, the upper portion in Fig. $3 b$ is predominated with higher concentrations of PL species, and lower portion in Fig. $3 \mathrm{~b}$ is predominated with lower concentrations of PL species. However, it is difficult to correlate PL concentrations to the geographical and clinical features of samples, due to limited sample size.

In male population, significant differences in PL concentrations between BHT and HCC were seen in 59 out of 320 individual PL species. Unlike in female population, there were more PL species significantly lower in HCC than in BHT in male population: among 59 individual PL species, 11 PL species were significantly increased, and 48 were statistically decreased in HCC than in BHT. The hierarchical cluster analysis (HCA) for the 59 species as shown in Fig. 3c revealed that the 59 individual PL species were distributed in 12 of 13 PL classes, except for PL class of PE-Cers. Among 12 classes, PC class had most individual species (16) significantly different between BHT and HCC. The rest 11 classes had varied number of individual species ranging 1 to 10 . Similarly, the upper portion in Fig. $3 \mathrm{c}$ is predominated with individual PL species having higher concentrations, and the lower portion in Fig. $3 \mathrm{c}$ is predominated with individual PL species having lower concentrations. The samples in upper portion have higher percentage of BHT (55\% vs. $50 \%$ ), and are more from patients with older age (69.8 vs. 63.9), lower percentage of HCC in late stage (11\% vs. $30 \%$ ), and higher percentage of HCC in higher grade (77.8 vs. $50 \%$ ), as compared to samples in lower portion.

Taken together, in all population and male population, more PL individual species were significantly decreased in HCC than in BHT, and these decreased PL species more related to younger male HCC patients in late stage, but not to HCC grade. On the contrary, in female population, more $\mathrm{PL}$ individual species were significantly increased in HCC than in BHT.

\section{Gender differences of phospholipids in class, in subgroup, and in total between BHT and HCC}

In the level of PL class, total ePE and total PE were significantly lower in HCC than in BHT in all population ( $p=0.0157$ and $p=0.0156$, respectively), and stratified male population ( $p=0.0149$ and $p=0.0073$, respectively). None of PL classes was statistically different between BHT and HCC in female population. While majority of PL classes were lower or unchanged in HCC than BHT, PE-Cer is the only PL class being obviously higher in $\mathrm{HCC}$ than in BHT in all studied populations, though the differences were not statistically significant.

PLs in all subgroups were lower in HCC than in BHT in all studied populations. However, significantly decreased PLs in HCC than in BHT was only seen in ethanolaminecontaining subgroup in all population $(p=0.0157)$ and stratified male population $(p=0.0076)$, but not in stratified female population.

As well, the concentration of total phospholipids was lower in HCC than in BHT in all studied populations. As compared to female population, male population had about $10 \%$ more decreased in HCC than in BHT. All differences were not statistically significant. Table 3 showed differences of PLs in class, in subgroup, and in total between BHT and HCC.

\section{Identification of PL biomarkers in the diagnosis and prognosis of HCC}

Lipid profiling revealed that a number of PLs in individual species, classes, and subgroups were significantly different between BHT and HCC. To further identify if these PLs can serve as biomarkers in differentiation HCC from BHT, the following criteria were set for selection: (1) PL concentration was higher than detection limit $(0.002 \mathrm{nmol} / \mathrm{mg} w w t)$ in at least one of BHT and HCC; (2) the differences in PL concentrations between BHT and HCC were statistically significant, no matter it is higher or lower in HCC; (3) all items of predicting power, especially sensitivity, specificity, and accuracy (AUC) were above 60\% simultaneously. The PLs that fulfilled these criteria were all PL individual species, including six in all population: ePC (34:2), ePC (36:2), PC (30:0), PC (34:2), PE (34:2), and SM (24:0); two in female population: PC (30:0) and PS (42:9); six in male population: PA (36:4), PC (30:0), PC (34:2), PE (34:3), PE (38:6), and PI (36:2), as shown in Fig. 4a. Among these biomarkers, PC 30:0 is the only biomarker common to all studied populations, and PC (34:2) is a common biomarker to all population and stratified male population. Four biomarkers are specific to all population, one to female population, and four to male population.

To evaluate if these biomarkers are also able to predict the progression of $\mathrm{HCC}$, these identified PL biomarkers in each study population were further correlated to clinical stage and pathological grade of HCC. ANOVA analysis indicated that all of the six identified PL biomarkers in all population significantly correlated to the progression of clinical stage (Fig. 4b) and pathological grade of HCC (Fig. 4c). In female population, only PC (30:0) did significantly correlate to the clinical stage and pathological grade of HCC (Fig. 4d). Except PI (36:2), the rest five PL biomarkers statistically correlated to the progression of clinical stage (Fig. 4e) and pathological grade (Fig. 4f) of HCC in male population.

Two of these identified biomarkers in PC class were unique. One was PC (30:0), which was the only identified PL biomarker that significantly higher in $\mathrm{HCC}$ than in BHT in all studied populations. Another one was PC (34:2), the most abundant PL species was lower in HCC than in BHT in all studied populations. Both increased PC (30:0) and decreased 

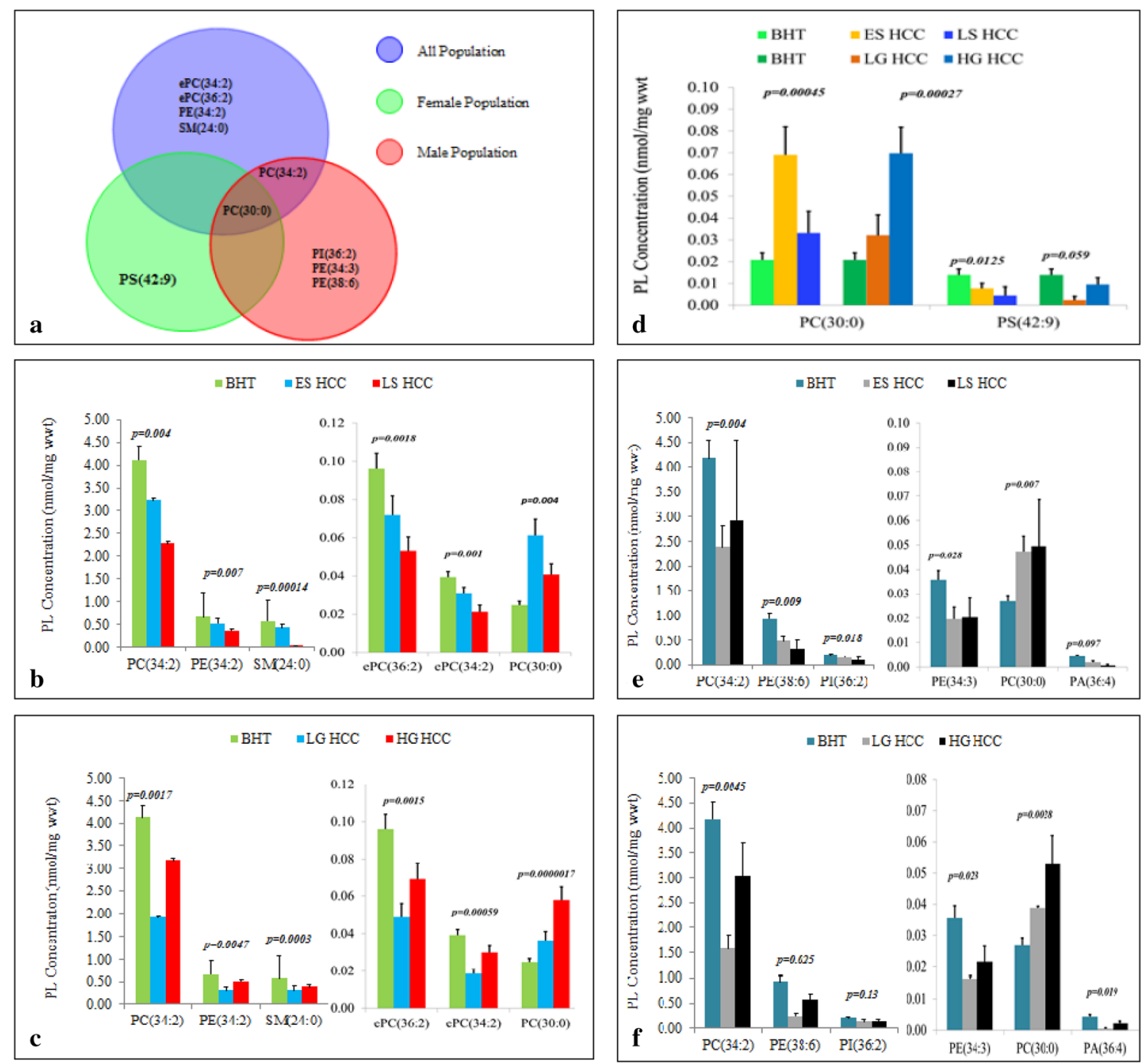

Fig. 4 Distribution of PL biomarkers among populations and their correlation to clinical progression of HCC. $B H T$ Benign hepatic tissues, $H C C$ hepatocellular carcinoma, ES early stage, $L S$ late stage, $L G$ low grade, $H G$ high grade. a The distribution of identified PL biomarkers in all studied populations; $\mathbf{b}$ correlation of identified PL biomarkers to clinical stage of HCC in all population; $\mathbf{c}$ correlation of identified PL biomarkers to pathological grade of HCC in all popula- tion; $\mathbf{d}$ correlation of identified PL biomarkers to clinical stage and pathological grade of HCC in female population; e correlation of identified PL biomarkers to clinical stage of HCC in male population; $\mathbf{f}$ correlation of identified PL biomarkers to pathological grade of HCC in male population. Data represent mean \pm standard error and $p<0.05$ was set statistical significance (ANOVA analysis)

In summary, all identified PL biomarkers and, especially, derived ratio of PC (34:2) to PC (30:0) are not only able to differentiate HCC from BHT with high sensitivity, specificity, and accuracy but also are able to predict the progression of HCC in terms of clinical stage and pathological grade. As compared to female population, male population had more gender-specific PL biomarkers in the differentiation of $\mathrm{HCC}$ from $\mathrm{BHT}$ and in the prediction of HCC progression. 
Table 4 Diagnostic power of the ratio of PC (34:2) to PC (30:0) in all studied populations
Table 5 Prognostic power of the ratio of PC (34:2) to PC (30:0) in all studied populations

\begin{tabular}{llllllllll}
\hline Population & & BHT & HCC & Sen & Spec & Prec & Recall & $F$-M & AUC \\
\hline All & $n$ & 32 & 32 & $87.50 \%$ & $93.75 \%$ & $93.33 \%$ & $87.50 \%$ & $90.32 \%$ & $94.43 \%$ \\
& Mean & 1.66 & 0.65 & & & & & & \\
& SD & 0.57 & 0.41 & & & & & & \\
& $p$ value & 0.000000000023 & & & & & & & \\
Female & $95 \%$ CI & $0.76-1.26$ & & & & & & & \\
& $n$ & 11 & 11 & $81.82 \%$ & $81.82 \%$ & $81.82 \%$ & $81.82 \%$ & $81.82 \%$ & $93.39 \%$ \\
& Mean & 1.85 & 0.65 & & & & & & \\
& SD & 0.69 & 0.39 & & & & & & \\
& $p$ value & 0.000067 & & & & & & & \\
Male & $95 \%$ CI & $0.70-1.69$ & & & & & & & \\
& $n$ & 21 & 21 & $90.48 \%$ & $95.24 \%$ & $95.00 \%$ & $90.48 \%$ & $92.68 \%$ & $95.24 \%$ \\
& Mean & 1.56 & 0.65 & & & & & & \\
& SD & 0.48 & 0.43 & & & & & & \\
& $p$ value & 0.00000011 & & & & & & & \\
& $95 \%$ CI & $0.62-1.19$ & & & & & & & \\
\hline
\end{tabular}

$B H T$ benign hepatic tissue, $H C C$ hepatocellular carcinoma, Sen sensitivity, Spec specificity, Prec precision; $F-M \mathrm{~F}$ measurement, $A U C$ area under curve

\begin{tabular}{|c|c|c|c|c|c|c|c|c|c|c|}
\hline \multirow[t]{2}{*}{ Population } & \multicolumn{3}{|c|}{ BHT } & \multicolumn{3}{|c|}{ ES HCC } & \multicolumn{3}{|c|}{ LS HCC } & \multirow[t]{2}{*}{$p$ value* } \\
\hline & $n$ & Mean & SD & $n$ & Mean & SD & $n$ & Mean & SD & \\
\hline \multicolumn{11}{|c|}{ HCC clinical stage } \\
\hline All & 32 & 1.66 & 0.57 & 12 & 0.62 & 0.34 & 16 & 0.69 & 0.51 & 0.0000000028 \\
\hline Female & 11 & 1.85 & 0.69 & 5 & 0.40 & 0.26 & 4 & 0.92 & 0.46 & 0.00057 \\
\hline Male & 21 & 1.56 & 0.48 & 15 & 0.59 & 0.31 & 4 & 0.97 & 0.78 & 0.0000014 \\
\hline \multicolumn{11}{|c|}{ HCC pathological grade } \\
\hline All & 32 & 1.66 & 0.57 & 11 & 0.61 & 0.39 & & 0.69 & 0.48 & 0.0000000028 \\
\hline Female & 11 & 1.85 & 0.69 & 4 & 0.92 & 0.46 & 5 & 0.40 & 0.26 & 0.00055 \\
\hline Male & 21 & 1.56 & 0.48 & 7 & 0.44 & 0.22 & 12 & 0.80 & 0.50 & 0.0000010045 \\
\hline
\end{tabular}

$B H T$ benign hepatic tissue, HCC hepatocellular carcinoma, ES HCC early-stage HCC, LS HCC late-stage HCC, $H G H C C$ high-grade HCC

$* p$ value was calculated by ANOVA

\section{Discussion}

This study first employed ESI-MS techniques in determining global PL profiles in paired BHT and HCC samples from same female and male patients. We found that (1) the concentrations of PLs in total, in most subgroups and classes, and in majority of individual species were lower in HCC than in BHT; (2) such decreases of PLs in HCC were more prominent in male patients than in female patients; (3) a panel of individual PL species can be used as diagnostic and prognostic biomarkers for HCC, either common to all population, or specific to stratified female and male populations.

Phospholipids are essential components of all cells. Previous studies suggested PLs increase with cell transformation and tumor progression in many cancers such as breast cancer and colorectal cancer cells $[11,12]$. None of the studies reported the differences in complete PL profiles between HCC and BHT samples. Few investigators performed partial PL profiles on plasma samples from HCC patients $[6,13,14]$. They found that several PL species were increased or decreased in plasma samples from HCC patients as compared to plasma samples from human subjects without cancers. Lu et al. also performed partial PL profiles on both hepatic tissues and sera simultaneously, and found that PL profiles in samples from sera and HCC tissues were not in agreement, implying that lipid metabolisms are likely to be modulated in different manners between HCC tissues and peripheral circulations [8]. Our results indicated that, unlike other cancers, HCC had lower concentrations of PLs in overall than that in 
BHT. This could be because of the facts that HCC cells are smaller in size than normal hepatocytes, and such reduction in HCC cell size becomes prominent in accordance with progressive thickening of tumor cell cords [15]. Actually, growth of HCC cells in smaller sizes are overnumbered HCC cells in larger size [16].

Each PL subgroup contains same head moiety. We found that the concentrations of PLs in all subgroups were lower in HCC. However, among PL subgroups, statistical difference was only seen in choline-containing subgroup between BHT and HCC in all population $(p=0.017)$ and stratified male population $(p=0.009)$. Phosphatidylcholines (PC) accounted $>93 \%$ choline-containing PLs (sphingomyelins were not included in this study) in both BHT and HCC in all studied populations. Decrease in PC and other cholinecontaining PLs in HCC might imply that the metabolism of these PLs favors degradation catalyzed by PC-specific phospholipase D in Kennedy's pathway. On the other hand, the expression level of choline kinase- $\alpha$, catalyzing the synthesis of phosphocholine is elevated in HCC [17] and other cancers. Thus, increase in degradation of choline-containing PLs and in the synthesis of phosphocholine might synergistically produce more phosphocholines in HCC tissues. Accumulated phosphocholines in HCC could serve as biomarkers in the diagnosis of $\mathrm{HCC}$ and in monitoring of therapeutic effects in the treatment of HCC by magnetic resonance spectroscopy imaging techniques, as demonstrated in HCC [18] and other cancer [19].

Ceramide phosphorylethanolamines (PE-Cer) are a group of sphingolipids that play critical roles in non-mammalian eukaryotes, similar to that of sphingomyelins in mammals [20]. Although it has been reported that mammalian cells also produce small amounts of the PE-Cers, the relationship of human PE-Cers with cancers have not been investigated. One study showed that PE-Cers isolated from Sphingobacterium spiritivorum ATCC 33861 can induce endonucleolytic DNA cleavage and apoptotic activity in human myeloid leukemia HL-60 cells in vitro [21]. PE-Cer was the least PL class, accounting for less than $0.01 \%$ in both BHT and HCC in all studied populations. Interestingly, PE-Cer was the only PL class with obvious higher concentration in HCC than in BHT in all studied populations. Further investigations are needed to clarify whether increase in PE-Cer produced in human HCC cells can also have similar functions in the induction of DNA damage and apoptosis against HCC cells seen in PE-Cers produced in bacteria on human myeloid leukemia HL-60 cells in vitro [21]. Perhaps it is a novel and potential therapeutic strategy to use exogenous PE-Cers in cancer treatment.

Effective therapies are feasible with surgical resection if HCC is diagnosed at an early stage [22]. However, the majority of patients are diagnosed at intermediate or advanced stage, resulting in high HCC-related deaths. Thus, identification of novel diagnostic and prognostic biomarkers is of great importance in early detection, monitoring therapy and predicting outcomes of HCC. To date, serum alpha-fetoprotein (AFP) remains the most commonly used biomarker in HCC. However, its use remains unsatisfactory and controversial, because AFP is only expressed in 60-80\% of HCC cases [23] and because its sensitivity and specificity are inadequate [24]. Currently, a plethora of new diagnostic and prognostic biomarkers for HCC are in development, but have not been widely used in practice [25]. In our study, as compared to AFP, all identified PL biomarkers in hepatic tissues possessed higher sensitivity, specificity, and accuracy in the differentiation of HCC from BHT. However, these results were obtained from a limited size sample, and need to be further validated in a large study cohort.

Interestingly, PC (30:0) was found to be more abundant in breast cancer area as compared to the stroma surrounding the cancer, and considered as abnormal lipid metabolismgenerated metabolites that promote cancer metastasis [26]. Kurabe et al. found that PC (34:2) was one of the few PC species highly downregulated in gastric cancer by imaging mass spectrometry (IMS) [27]. In agreement with these studies, we found that increased PC (30:0) and decreased PC (34:2) in HCC were able to differentiate HCC from BHT with high sensitivity, specificity, and accuracy simultaneously while they were used alone. When the ratio of PC (30:0) to PC (34:2) was used, the powers in differentiating HCC from BHT (Table 4) and in predicting clinical progression of HCC (Table 5) were dramatically amplified. Of course, these PL biomarkers need to be further validated in larger study cohorts as well.

The incidence, morbidity, and mortality rates of HCC are obviously disparate between male and female populations [28, 29]. The molecular mechanisms underlying these gender disparities have not been fully elucidated yet. Given that alterations in PLs between BHT and HCC correlate with occurrence, progression and clinical outcomes of HCC, gender differences in PL alterations should be in parallel reflected to these disparities. As compared to female HCC patients, male HCC patients had lower HCC to BHT ratio of total PLs ( 0.80 in male and 0.89 in female), more PL individual species significantly decreased in HCC than in BHT (59 vs.7), and more gender-specific PL biomarkers identified (4 vs. 1). Also, the identified male-specific PL biomarkers had relatively higher power in the differentiation of HCC from BHT, and in the prediction of clinical progression of HCC than female-specific PL biomarkers. In addition, male HCC patients had lower concentrations of PER-Cer in HCC than in BHT, although it is not sure yet that increasing in PE-Cer is of protective effect on the oncogenesis of HCC. Overall, differences in the alterations of PL in HCC between female and male are mirrored gender disparities in epidemiological and clinical manifestations of HCC. 
Male gender, onset of HCC at younger age, late clinical stage, and higher tumor grade are all associated with poor prognoses of HCC. In agreement, our study indeed revealed that decreased hepatic PLs were more related to male sex, younger age at onset, and HCC in late stage, but not to HCC grade. This could be due to the fact that only 2 out of 32 cases were classified as Grade III, and the majority of cases were classified grade I and grade II, according to the Edmondson-Steiner Classification of HCC.

\section{Conclusions}

In conclusion, the findings in this study could provide new evidence in understanding the roles of PLs in oncogenesis, progression and gender disparities, in exploring new PL biomarkers in diagnosis and prognosis, and in discovering novel therapeutic targets for HCC.

Acknowledgements We would like to thank Mary Roth, Libin Yao, and Ruth Welti of the Kansas Lipidomic Research Center for lipid analysis. Instrument acquisition and lipidomics method development was supported by the National Science Foundation, K-IDeA Networks of Biomedical Research Excellence (INBRE) of National Institute of Health (P20GM103418), and Kansas State University.

Author contributions Formal analysis: ZL and HM; resources: ZZ and JM; data curation: ZZ; software: HM; writing, original draft preparation: ZL; writing, review and editing: JM; supervision and project administration: XZ

\section{Compliance with ethical standards}

Conflict of interest Zhirong Liu, Zhen Zhang, Hao Mei, Jinghe Mao, Xinchun Zhou declare no conflict of interest.

Ethical approval This study was approved by Institutional Review Board of University of Mississippi Medical Center.

Informed consent No consent form was needed.

Open Access This article is licensed under a Creative Commons Attribution 4.0 International License, which permits use, sharing, adaptation, distribution and reproduction in any medium or format, as long as you give appropriate credit to the original author(s) and the source, provide a link to the Creative Commons licence, and indicate if changes were made. The images or other third party material in this article are included in the article's Creative Commons licence, unless indicated otherwise in a credit line to the material. If material is not included in the article's Creative Commons licence and your intended use is not permitted by statutory regulation or exceeds the permitted use, you will need to obtain permission directly from the copyright holder. To view a copy of this licence, visit http://creativecommons.org/licenses/by/4.0/.

\section{References}

1. Ferlay J, Soerjomataram I, Dikshit R, Eser S, Mathers C, Rebelo M, Parkin DM, Forman D, Bray F. Cancer incidence and mortality worldwide: sources, methods and major patterns in GLOBOCAN 2012. Int J Cancer 2015;136:E359-E386386

2. Omata M, Cheng AL, Kokudo N, Kudo M, Lee JM, Jia J, Tateishi R, Han KH, Chawla YK, Shiina S, et al. Asia-Pacific clinical practice guidelines on the management of hepatocellular carcinoma: a 2017 update. Hepatol Int 2017;11:317-370

3. Lydic TA, Goo YH. Lipidomics unveils the complexity of the lipidome in metabolic diseases. Clin Transl Med 2018;7:4

4. Dill AL, Eberlin LS, Zheng C, Costa AB, Ifa DR, Cheng L, Masterson TA, Koch MO, Vitek O, Cooks RG. Multivariate statistical differentiation of renal cell carcinomas based on lipidomic analysis by ambient ionization imaging mass spectrometry. Anal Bioanal Chem 2010;398:2969-2678

5. Zhou X, Mao J, Ai J, Deng Y, Roth MR, Pound C, Henegar J, Welti R, Bigler SA. Identification of plasma lipid biomarkers for prostate cancer by lipidomics and bioinformatics. PLoS One 2012;7:e48889

6. Cotte AK, Cottet V, Aires V, Mouillot T, Rizk M, Vinault S, Binquet C, Barros J-PPD, Hillon P, Delmas D. Phospholipid profiles and hepatocellular carcinoma risk and prognosis in cirrhotic patients. Oncotarget 2019;10:2161-2172

7. Krautbauer S, Meier EM, Rein-Fischboeck L, Pohl R, Weiss TS, Sigruener A, Aslanidis C, Liebisch G, Buechler C. Ceramide and polyunsaturated phospholipids are strongly reduced in human hepatocellular carcinoma. Biochim Biophys Acta 2016;1861:1767-1774

8. Lu Y, Chen S, Chen J, Yu K, Huang C, Zhang QL, Chen M, Ong $\mathrm{CN}$. Comparison of hepatic and serum lipid signatures in hepatocellular carcinoma patients leads to the discovery of diagnostic and prognostic biomarkers. Oncotarget 2018;9:5032-5043

9. Bligh EG, Dyer WJ. A rapid method of total lipid extraction and purification. Can J Biochem Physiol 1959;37:911-917

10. Xu J, Chen Y, Zhang R, Song Y, Cao J, Bi N, Wang J, He J, Bai $\mathrm{J}$, Dong L, et al. Global and targeted metabolomics of esophageal squamous cell carcinoma discovers potential diagnostic and therapeutic biomarkers. Mol Cell Proteom 2013;12:1306-1318

11. Sakai K, Okuyama H, Yura J, Takeyama H, Shinagawa N, Tsuruga N, Kato K, Miura K, Kawase K, Tsujimura T, et al. Composition and turnover of phospholipids and neutral lipids in human breast cancer and reference tissues. Carcinogenesis 1992;13:579-584

12. Dobrzy’nska I. Barbara Szachowicz-Petelska; Sulkowski, S. 1.; Figaszewski, Z. Changes in electric charge and phospholipids composition in human colorectal cancer cells. Mol Cell Biochem 2005;276:113-119

13. Lu Y, Chen J, Huang C, Li N, Zou L, Chia SE, Chen S, Yu K, Ling Q, Cheng Q, et al. Comparison of hepatic and serum lipid signatures in hepatocellular carcinoma patients leads to the discovery of diagnostic and prognostic biomarkers. Oncotarget 2018;9:5032-5043

14. Krautbauer S, Weiss TS, Wiest R, Schacherer D, Liebisch G, Buechler C. Diagnostic value of systemic cholesteryl ester/free cholesterol ratio in hepatocellular carcinoma. Anticancer Res 2017;37:3527-3535

15. Kondo F, Wada K, Kondo Y. Morphometric analysis of hepatocellular carcinoma. Virchows Archiv A Pathol Anat 1988;413:425-430

16. Amodeo AA, Skotheim JM. Cell-Size Control. Cold Spring Harb Perspect Biol 2016;8:a019083

17. Lin XM, Hu L, Gu J, Wang RY, Li L, Tang J, Zhang BH, Yan $\mathrm{XZ}$, Zhu YJ, Hu CL, et al. Choline kinase $\alpha$ mediates interactions 
between the epidermal growth factor receptor and mechanistic target of rapamycin complex 2 in hepatocellular carcinoma cells to promote drug resistance and xenograft tumor progression. Gastroenterology 2017;152:1187-1202

18. Yang Y, Li C, Nie X, Feng X, Chen W, Yue Y, Tang H, Deng F. Metabonomic studies of human hepatocellular carcinoma using high-resolution magic-angle spinning 1H NMR spectroscopy in conjunction with multivariate data analysis. J Proteome Res 2007;6:2605-2614

19. Glunde K, Jie C, Bhujwalla ZM. Molecular causes of the aberrant choline phospholipid metabolism in breast cancer. Cancer Res 2004;64:4270-4276

20. Masood MA, Yuan C, Acharya JK, Veenstra TD, Blonder J. Quantitation of ceramide phosphorylethanolamines containing saturated and unsaturated sphingoid base cores. Anal Biochem 2010;400:259-269

21. Minamino M, Sakaguchi I, Naka T, Ikeda N, Kato Y, Tomiyasu I, Yano I, Kobayashi K. Bacterial ceramides and sphingophospholipids induce apoptosis of human leukaemic cells. Microbiology 2003;149:2071-2081

22. Forner A, Reig M, Bruix J. Hepatocellular carcinoma. Lancet 2018;391:1301-1304

23. Galle PR, Foerster F, Kudo M, Chan SL, Llovet JM, Qin S, Schelman W, Chintharlapalli S, Abada P, Sherman M, et al. Biology and significance of alpha-fetoprotein in hepatocellular carcinoma. Liver Int 2019;39:2214-2229

24. European Association for Study of, L.; European Organisation for, R.; Treatment of, C. EASL-EORTC clinical practice guidelines: management of hepatocellular carcinoma. Eur J Cancer 2012;48:599-641

25. Lou J, Zhang L, Lv S, Zhang C, Jiang S. Biomarkers for hepatocellular carcinoma. Biomark Cancer 2017;9:1-9

26. Hosokawa Y, Masaki N, Takei S, Horikawa M, Matsushita S, Sugiyama E, Ogura H, Shiiya N, Setou M. Recurrent triple-negative breast cancer (TNBC) tissues contain a higher amount of phosphatidylcholine (32:1) than non-recurrent TNBC tissues. PLoS One 2017;12:e0183724

27. Kurabe N, Suzuki M, Inoue Y, Kahyo T, Iwaizumi M, Konno H, Setou M, Sugimura H. Abstract 394A: Phosphatidylcholine-34:2 and $-36: 4$ have tumor suppressive function for gastric cancer. Can Res 2016;76:394A

28. Wu Y, Yao N, Feng Y, Tian Z, Yang Y, Zhao Y. Identification and characterization of sexual dimorphismlinked gene expression profile in hepatocellular carcinoma. Oncol Rep 2019;42:937-952

29. Lee CM, Lu SN, Changchien CS, Yeh CT, Hsu TT, Tang JH, Wang JH, Lin DY, Chen CL, Chen WJ. Age, gender, and local geographic variations of viral etiology of hepatocellular carcinoma in a hyperendemic area for hepatitis B virus infection. Cancer 1999;86:1143-1150

Publisher's Note Springer Nature remains neutral with regard to jurisdictional claims in published maps and institutional affiliations. 